\title{
Near-infrared speckle interferometry and radiative transfer modelling of the carbon star LP Andromedae ${ }^{\star}$
}

\author{
A. B. Men'shchikov ${ }^{1,2}$, Y. Y. Balega ${ }^{3}$, M. Berger ${ }^{2}$, T. Driebe ${ }^{2}$, K.-H. Hofmann ${ }^{2}$, \\ A. F. Maximov ${ }^{3}$, D. Schertl ${ }^{2}$, V. I. Shenavrin ${ }^{4}$, and G. Weigelt ${ }^{2}$ \\ ${ }^{1}$ Institute for Computational Astrophysics, Saint Mary's University, Halifax, NS B3H 3C3, Canada \\ e-mail: amenshch@ap.stmarys.ca \\ 2 Max-Planck-Institut für Radioastronomie, Auf dem Hügel 69, 53121, Bonn, Germany \\ 3 Special Astrophysical Observatory, Nizhnij Arkhyz, Zelenchuk region, Karachai-Cherkesia, 35147, Russia \\ 4 Sternberg Astronomical Institute, Universitetskii Prosp. 13, 119899 Moscow, Russia
}

Received 3 March 2005 / Accepted 5 October 2005

\section{ABSTRACT}

\begin{abstract}
We present the near-infrared speckle interferometry for LP And in the $H$ and $K^{\prime}$ bands with diffraction-limited resolutions of 56 and 72 mas, new JHKLM photometry, and the results of our radiative transfer modelling of this carbon star. The reconstructed visibility reveals a sphericallysymmetric envelope surrounding the central star. To determine the physical parameters of the latter and the properties of its dusty envelope, we performed extensive radiative transfer calculations. The well-defined spectral energy distribution of LP And in the entire range from the near-IR to millimeter wavelengths (including the absorption feature visible in the stellar continuum at $3 \mu \mathrm{m}$ and the shapes of the dust emission bands at 11 and $27 \mu \mathrm{m}$ ), together with our $H$-band visibility can be reproduced by a spherical dust envelope with parameters that are very similar to those of CW Leo (IRC+10216), the best studied carbon star. For the newly estimated pulsation period $P=617 \pm 6$ days and distance $D=740 \pm 100 \mathrm{pc}$, our model of LP And changes its luminosity $L_{\star}$ between 16200 and $2900 L_{\odot}$, its effective temperature $T_{\star}$ between 3550 and $2100 \mathrm{~K}$, and its radius $R_{\star}$ between 340 and $410 R_{\odot}$. The model estimates the star's mass-loss rate $\dot{M} \approx 1.9 \times 10^{-5} M_{\odot} \mathrm{yr}^{-1}$, assuming a constant outflow velocity $v=14 \mathrm{~km} \mathrm{~s}^{-1}$. If the latter also applied to the innermost parts of the dusty envelope, then presently the star would be losing mass at a rate $\dot{M} \approx 6.0 \times 10^{-5} M_{\odot} \mathrm{yr}^{-1}$. However, we believe that the inner wind velocity must actually be closer to $v \approx 4 \mathrm{~km} \mathrm{~s}^{-1}$ instead, as wind acceleration is expected in the dust-formation zone. The dusty envelope of LP And extends from $R_{1} \approx 2 R_{\star}$ to distances of $R_{2} \approx 3$ pc from the star. The total mass of the envelope lost by the central star is $M=3.2 M_{\odot}$ assuming a dust-to-gas mass ratio of $\rho_{\mathrm{d}} / \rho=0.0039$. The circumstellar optical depth towards the star is $\tau_{V}=25$ in the visual. The dust model contains small silicon carbide grains, inhomogeneous grains made of a mixture of $\mathrm{SiC}$ and incompletely amorphous carbon, and thin mantles made of iron-magnesium sulfides. This dust mixture perfectly fits the infrared continuum and both the $11.3 \mu \mathrm{m}$ and $27 \mu \mathrm{m}$ emission bands. We find that our $K^{\prime}$-band visibility could not be fitted by our spherical model, so we discuss possible reasons for this interesting result. More observations are required in order to determine what causes this effect. If slight deviations from spherical geometry in its envelope are the reason, then the object's evolutionary stage would be even more similar to that of CW Leo. It appears that LP And is a highly-evolved intermediate-mass star (initial mass $M_{\star}^{0} \approx 4 M_{\odot}$ ) at the end of its AGB phase.
\end{abstract}

Key words. radiative transfer - stars: circumstellar matter - stars: AGB and post-AGB - stars: mass-loss - stars: individual: LP Andromedae - techniques: image processing

\section{Introduction}

The carbon star LP And, also known as RAFGL 3116, IRC +40540 , and IRAS 23320+4316, has the spectral type C VIII 3.5e (Cohen 1979). Carbon stars form a subclass of those evolved stars on the asymptotic giant branch (AGB) that have atmospheres enriched in carbon, produce large amounts of carbonaceous dust, and supply the interstellar medium with both heavy elements and dust grains. Such stars are variable,

* Based on data collected at the $6 \mathrm{~m}$ telescope of the Special Astrophysical Observatory and the $1.25 \mathrm{~m}$ telescope of the Crimean Astrophysical Observatory. pulsating with periods $100 \lesssim P \lesssim 1000$ days, and have high mass-loss rates and thus relatively massive and opaque dusty circumstellar envelopes. As the envelopes are optically thick, they reprocess the optical radiation of the central star into the infrared, where they are very bright. The best-studied object of this type is the carbon star CW Leo, also known as IRC+10 216 (see, e.g., Men'shchikov et al. 2001, 2002a; Weigelt et al. 2002, and references therein), whose many properties are remarkably similar to those of LP And, as it will be shown in this paper.

Fitting photometric data of LP And in the $L$ band from October 1979 to December 1988, Jones et al. (1990) derived its pulsation period of $P \approx 620$ days. Using observations 
in $\mathrm{H}_{\alpha}$ and $\mathrm{NaD}$ lines, as well as in 3 optical bands at 0.52 , 0.56 , and $0.66 \mu \mathrm{m}$ from July 1977 to July 1981, Cohen \& Hitchon (1996) derived a period $P=628 \pm 26$ days. With this value, Lindqvist et al. (2000) estimated an average luminosity $\left\langle L_{\star}\right\rangle \approx 9400 L_{\odot}$ using the period-luminosity relation for Mira stars (Groenewegen \& Whitelock 1996). They also fitted the SED of LP And using a superposition of 2 blackbodies to determine the bolometric flux and estimate a distance of $D \approx 630 \mathrm{pc}$. Using measurements of CO lines, Schöier \& Olofsson (2001) determined a mass-loss rate $\dot{M} \approx 1.5 \times 10^{-5} M_{\odot} \mathrm{yr}^{-1}$ and an outflow velocity $v \approx 14.0 \mathrm{~km} \mathrm{~s}^{-1}$.

\section{Observational data}

The $H$ - and $K^{\prime}$-band speckle interferograms of LP And were obtained with the $6 \mathrm{~m}$ telescope of the Special Astrophysical Observatory (SAO) in Russia on September 22-23, 2002 and October 12, 2003 (Table 1). The data were recorded in the primary focus of the telescope with our $512 \times 512$ pixel HAWAII speckle camera through interference filters. Additional speckle interferograms were taken for three unresolved reference stars (HD 222018, HD 221516, and BD +424696). The $H$ - and $K^{\prime}$-band visibilities were derived from the speckle interferograms using the speckle interferometry method (Labeyrie 1970).

The reconstructed 2D visibilities of LP And in the $H$ and $K^{\prime}$ bands, together with the azimuthally averaged visibility profiles are shown in Fig. 1. Within the observational uncertainties, the $K^{\prime}$ data from 2003 are the same as those obtained a year before. We would like to emphasize that, within the observational uncertainties, no deviations from spherical symmetry can be found in the $2 \mathrm{D}$ visibilities, although one could perhaps see some structures in Fig. 1. This can be verified by using different subsets of the raw data and considering azimuthal variations in the power spectra.

The averaged visibilities decrease steadily to below 0.5 at the diffraction cut-off frequencies. Thus, the envelope of LP And is resolved, and the contribution of the unresolved component to the monochromatic fluxes is $\sim 40 \%$, which indicates rather large optical depths at these wavelengths. In order to give first rough estimates for the diameter of the dusty envelope, the azimuthally averaged visibility were fitted with Gaussian and uniform disc models describing the center-tolimb brightness variation (see Table 1, Fig. 1). The shape of the observed visibility strongly deviates from those functions, and it is obvious that both intensity distributions and diameters have no physical meaning. Much more realistic physical parameters of the envelope of LP And are reconstructed by our radiative transfer modelling (see Sect. 3).

\subsection{Pulsation period}

In this study, we attempted to obtain a more accurate estimate of the pulsation period of LP And using a larger time interval over 25 years. For this purpose, we utilized the $K$-band photometry published by Jones et al. (1990) (obtained between October 1979 and December 1988), $K$ band ISO SWS flux (December 1996), unpublished $K$-band photometry by Boris Yudin (August 2003 to November 2003), and our new $K$-band fluxes (December 2003 to December 2004, Table 2). The photometry in 2003-2004 was obtained at the Crimean Astrophysical Observatory. The photometric data and a least-squares fit to the light curve are shown in Fig. 2. The plot also indicates the dates of our speckle-interferometry observations and of the ISO SWS spectrophotometry of LP And; this information will be used later to link the observations to the photometric phases.

The fit shown in Fig. 2 corresponds to a period $P=617 \pm$ 2 days. Since in addition to the small statistical error of 2 days, there may be larger systematic errors in the period, we conservatively estimate its uncertainty as $\sim 1 \%$ and adopt the period of $P=617 \pm 6$ days in this work.

\subsection{Spectral energy distribution}

To utilize all the information coded in the spectral energy distribution (SED) of an object, it is very important to collect as much photometric data as possible. LP And has a very well-defined infrared SED in the $2-200 \mu \mathrm{m}$ wavelength range that contains its peak at $11 \mu \mathrm{m}$, thanks to the ISO spectrophotometry (see Fig. 3). Using the online Catalog of Infrared Observations (Gezari et al. 1999), we found many other additional fluxes for LP And published in the literature (Lockwood 1974; Strecker \& Ney 1974; Simon 1974; Gullixson et al. 1983; Cohen 1984; Jones et al. 1990; Margulis et al. 1990; Groenewegen et al. 1993). In addition, we also used our new observations of LP And in the $J, H, K, L$, and $M$ bands (Table 2).

The data were obtained at different epochs and with different instruments. Therefore, it is not surprising that at each wavelength below $20 \mu \mathrm{m}$ those fluxes are scattered by roughly a factor of 3-10 (Figs. 3, 4). This scatter reflects not only the periodic and non-periodic variability of the star, but also different accuracies and possibly different apertures used in the measurements. Also it may well be an indication that there are some deviations in the envelope's density distribution from spherical symmetry.

\subsection{Bolometric fluxes}

We can use the ISO spectrum and add the other photometric data outside its wavelength range to estimate the bolometric flux of LP And. However, it is difficult to find enough measurements at the phase of the ISO SWS observations $(\phi \approx 0.64)$. Since the ISO spectrum is reasonably close to the minimum brightness of LP And, we chose to use a lower boundary of all other photometric points as the extrapolation of the ISO's SED towards $1 \mu \mathrm{m}$ and $1.1 \mathrm{~mm}$. An integration of the resulting energy distribution gives an estimate of the bolometric flux $F_{0.64}=3.1 \times 10^{-10} \mathrm{~W} \mathrm{~m}^{-2}, 96 \%$ of which comes in the spectral region of the ISO observations $(2.4-197 \mu \mathrm{m})$.

In order to estimate the bolometric flux of LP And at the maximum of its brightness $(\phi=0.0)$, we used the upper boundary of the scattered fluxes at $1-18 \mu \mathrm{m}$, linearly interpolating between them; there are only very few measurements at 
Table 1. Observational parameters for LP And. Here, $\phi$ is the photometric phase $(0.5$ for minimum brightness on JD $=2450331)$ from the $K$-band light curve with $P=617$ days (Fig. 2), $\lambda_{\mathrm{c}}$ the central wavelength and $\Delta \lambda$ the FWHM bandwidth of the filters, $N_{\star}$ and $N_{\mathrm{R}}$ are the numbers of speckle interferograms for LP And and the reference stars, respectively, $T$ is the exposure time per frame, $S$ the seeing (FWHM), $p$ the pixel size, $R$ the resolution, and $d_{\mathrm{G}}$ and $d_{\mathrm{UD}}$ are the angular diameters (FWHM) of the source, based on fitting the Gaussian and uniform disc models to the azimuthally averaged visibilities.

\begin{tabular}{|c|c|c|c|c|c|c|c|c|c|c|c|c|c|c|}
\hline Filter & Date & Julian date & $\bar{\phi}$ & $\begin{array}{c}\lambda_{\mathrm{c}} \\
\mu \mathrm{m}\end{array}$ & $\begin{array}{l}\Delta \lambda \\
\mu \mathrm{m}\end{array}$ & $\overline{N_{\star}}$ & $N_{\mathrm{R}}$ & $\begin{array}{c}T \\
\mathrm{~ms}\end{array}$ & $\begin{array}{l}S \\
\prime \prime\end{array}$ & $\begin{array}{c}p \\
\text { mas }\end{array}$ & $\begin{array}{c}R \\
\text { mas }\end{array}$ & $\begin{array}{c}d_{\mathrm{G}} \\
\mathrm{mas}\end{array}$ & $\begin{array}{l}d_{\mathrm{UD}} \\
\operatorname{mas}\end{array}$ & Ref. star \\
\hline$H$ & Sep. 23, 2002 & 2452541 & 0.082 & 1.65 & 0.32 & 1050 & 1400 & 164 & 1.52 & 20.2 & 56 & 36.8 & 57.7 & HD 221516 \\
\hline \multirow[t]{2}{*}{$K^{\prime}$} & Sep. 22, 2002 & 2452540 & 0.080 & 2.12 & 0.21 & 2430 & 2100 & 164 & 1.76 & 27.0 & 72 & 39.2 & 62.7 & HD 222018 \\
\hline & Oct. 12,2003 & 2452925 & 0.704 & 2.12 & 0.21 & 800 & 800 & 41 & 2.00 & 28.7 & 72 & 34.1 & 55.1 & $\mathrm{BD}+424696$ \\
\hline
\end{tabular}
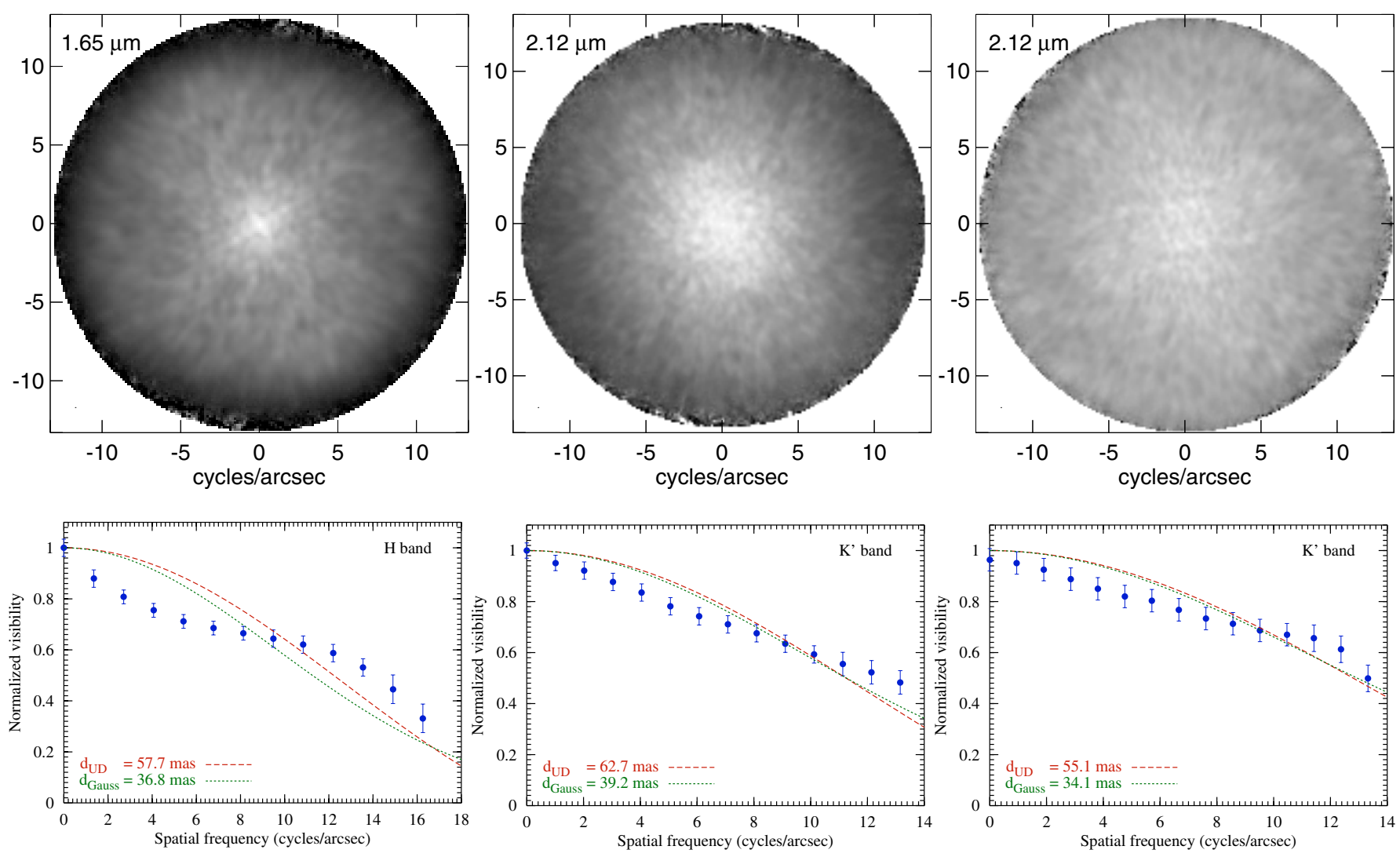

Fig. 1. Power spectra (upper panels) and azimuthally averaged visibilities (lower panels) of LP And in the $H$ and $K^{\prime}$ bands obtained in speckleinterferometry observations with the $6 \mathrm{~m}$ telescope at SAO (right panels show our latest results of 2003). No deviations from spherical symmetry are visible in the data. Shapes of the visibilities in $H$ and $K^{\prime}$ are different, suggesting a somewhat larger contribution of scattering by the dust grains in the $H$ band.

longer wavelengths. To obtain the maximum SED from $18 \mu \mathrm{m}$ to $1.1 \mathrm{~cm}$, we multiplied the extrapolated ISO SED (see above) by the ratio of the maximum flux at $\lambda=18 \mu \mathrm{m}$ to the ISO flux at the same wavelength. Using this approach, we obtained the integrated bolometric flux $F_{0.0}=9.5 \times 10^{-10} \mathrm{~W} \mathrm{~m}^{-2}$.

This reconstruction of the observed SED takes the strong variability of LP And into account, in contrast to previous estimates. Our method may not be very accurate, however, as the shape of the SED is also likely to vary when the stellar parameters change between the minimum and maximum phases. On the other hand, since the peak wavelength of the SED of
LP And is much shorter than the extrapolated SED ( $\sim 5 \mu \mathrm{m}$ vs. $\lambda>18 \mu \mathrm{m})$, the errors of our approach should not be large.

Since we have the bolometric fluxes of LP And at $\phi=0.64$ and 0.0 , one can assume its sinusoidal variation to obtain the bolometric flux $F_{0.5}=1.7 \times 10^{-10} \mathrm{~W} \mathrm{~m}^{-2}$ at minimum phase, a factor 5.6 lower than in the maximum of its brightness. One can approximate the flux variation by the following formula:

$F(t)=\left[5.58-3.89 \cos \left(2 \pi\left(t-t_{0.5}\right) / P\right)\right] \times 10^{-10} \mathrm{~W} \mathrm{~m}^{-2}$

where time $t$ is expressed in Julian days $\left(t_{0.5}=2450331\right)$ and the pulsation period $P$ is in days. 
Table 2. New $K$ and JHKLM photometry of LP And: date, Julian date, phase, and observed fluxes in the near-IR bands (in magnitudes).

\begin{tabular}{cccccccc}
\hline \hline Date & JD & $\phi$ & $J$ & $H$ & $K$ & $L$ & $M$ \\
\hline Aug. 15, 2003 & 2452867 & 0.610 & & 4.23 & & \\
Sep. 12, 2003 & 2452895 & 0.656 & & & 4.05 & & \\
Nov. 17, 2003 & 2452961 & 0.763 & & & 3.32 & & \\
Dec. 10, 2003 & 2452984 & 0.800 & & & 3.21 & & \\
Jan. 17, 2004 & 2453022 & 0.861 & & & 3.08 & & \\
Jul. 05, 2004 & 2453192 & 0.137 & & & 3.22 & & \\
Jul. 30, 2004 & 2453217 & 0.177 & & & 3.32 & & \\
Oct. 15, 2004 & 2453284 & 0.286 & & & 3.71 & & \\
Oct. 26, 2004 & 2453305 & 0.320 & 8.86 & 6.31 & 3.85 & 0.74 & -0.32 \\
Dec. 15, 2004 & 2453355 & 0.401 & 9.23 & 6.57 & 4.12 & 1.03 & 0.12 \\
\hline
\end{tabular}

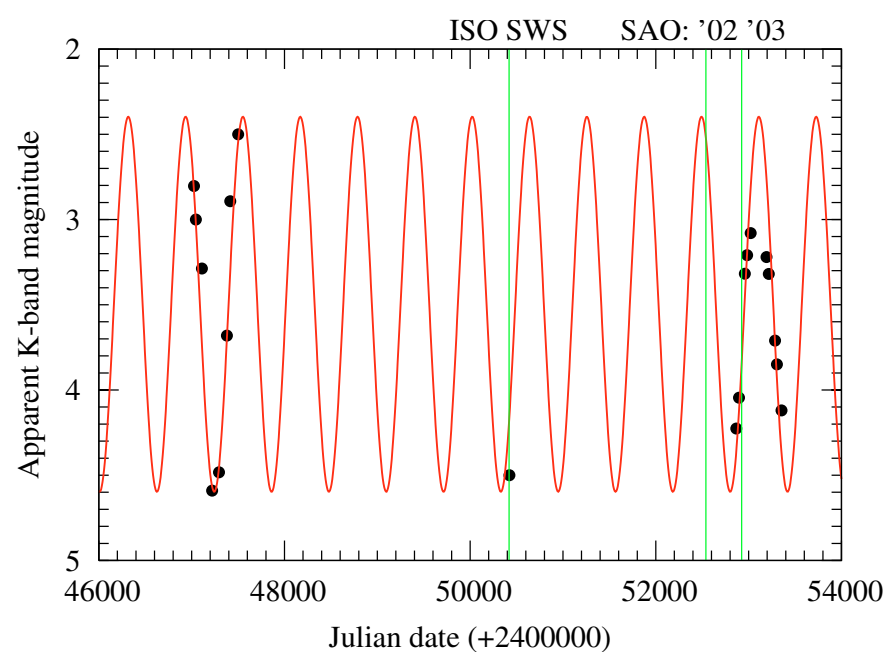

Fig. 2. Light curve of LP And in the $K$ band and its least-squares fit that gives a pulsation period $P=617$ days. Dates of the ISO SWS and our speckle-interferometry observations are indicated by the thin vertical lines.

\subsection{Luminosity and distance}

The pulsation period and bolometric flux can be used to derive both the luminosity of LP And and its distance. The periodluminosity relation of Groenewegen \& Whitelock (1996)

$M_{\mathrm{bol}}=-2.59^{\mathrm{m}} \log P+2.02^{\mathrm{m}}( \pm 0.26)$

can be applied, although with a bit of extrapolation to the longer pulsation period of LP And. We see, however, no reason to expect a sudden large change in the relationship for the $19 \%$ longer period than for the longest one for which the periodluminosity relation was derived.

With $P=617$ days, we obtain the absolute bolometric magnitude $\left\langle M_{\text {bol }}\right\rangle=-5.21^{\mathrm{m}}( \pm 0.26)$ and its average (over the pulsation cycle) luminosity $\left\langle L_{\star}\right\rangle=9550 L_{\odot}$ with the uncertainties of approximately $\pm 25 \%$. Using the average flux $\langle F\rangle=$ $5.6 \times 10^{-10} \mathrm{~W} \mathrm{~m}^{-2}$ from Eq. (1), we can estimate the distance $D=740$ pc with an uncertainty of $\sim 100$ pc (or $\pm 14 \%$ ), which has been adopted in this study.
Based on the above estimates, we can obtain the pulsation phases, bolometric fluxes, and luminosities of LP And corresponding to the dates of all observations (Table 3).

\section{The radiative transfer model}

The SED of LP And, including the shapes of its features at 11 and $27 \mu \mathrm{m}$, is remarkably similar to that of CW Leo, the beststudied long-period carbon star (see Fig. 3). Not only are the SEDs of the stars almost identical, but other parameters, such as their pulsation periods, luminosities, effective temperatures, masses, mass-loss rates, outflow velocities, and evolutionary stages (both are carbon stars) are also very similar. Thus, our idea was to use the detailed dust envelope model of CW Leo presented by Men'shchikov et al. $(2001,2002 \mathrm{a})$ as the basis for our numerical study of LP And. We refer to the papers for a more detailed description of the model geometry, dust properties, and modelling approach, as well as for a discussion of all assumptions.

We utilized our 2D dust continuum radiative transfer code (Men'shchikov \& Henning 1997). It is based on a ray-tracing method which provides an accurate solution to the frequencydependent radiative transfer problem, including isotropic scattering, in both spherical and simple axially-symmetric geometries. Large parameter space is carefully explored in hundreds of runs by changing model parameters and comparing the model observables with all available observational constraints.

Our model describes only the present structure of the envelope and, as such, is time-independent. The complex molecular chemistry of the envelope is largely ignored here, while the gas component is described by means of a spatially constant dust-to-gas mass ratio. The assumed constancy of $\rho_{\mathrm{d}} / \rho$ may not be realistic enough, thus contributing to the uncertainties of the derived properties of the gaseous envelope (e.g., the total mass $M$ of the envelope, lost by the central star during its evolution). With this in mind, we present our estimate of the gas mass in the envelope, since it is an important physical parameter.

Here we briefly summarize our general simplifying assumptions relevant to our modelling: (1) the dust envelope is spherically-symmetric; (2) the dust population consists of spherical, compact solid grains; (3) the size distribution of the grains can be described by a power law; (4) wherever a dust component exists, its composition, structure, and grain sizes are spatially invariant; (5) light scattering by the dust particles is isotropic; (6) dust-to-gas mass ratio of a dust component is spatially homogeneous; (7) dust is in radiative equilibrium with the radiation field; (8) the contribution of molecular line emission to the observed fluxes is small; (9) the star can be described by hydrostatic model atmospheres, and it radiates as a blackbody at long wavelengths.

There are no good reasons to believe that the above statements are extremely good approximations. One cannot avoid using them, however, primarily because of the obvious lack of sufficiently detailed and reliable observational constraints. 
Table 3. Derived bolometric flux $F$ and luminosity $L_{\star}$ of LP And as a function of its pulsation phase $\phi$ for the dates of key observations.

\begin{tabular}{lcccr}
\hline \hline Observations & Julian dates & $\phi$ & $\begin{array}{c}F(\phi) \\
10^{-10} \mathrm{~W} \mathrm{~m}^{-2}\end{array}$ & $\begin{array}{r}L_{\star}(\phi) \\
L_{\odot}\end{array}$ \\
\hline ISO SWS & 2450420 & 0.644 & 3.18 & 5440 \\
ISO LWS & 2450480 & 0.741 & 5.37 & 9190 \\
SAO H (2002) & 2452541 & 0.082 & 8.97 & 15400 \\
SAO $K^{\prime}(2002)$ & 2452540 & 0.080 & 8.99 & 15400 \\
SAO $K^{\prime}(2003)$ & 2452925 & 0.704 & 4.48 & 7660 \\
\hline
\end{tabular}

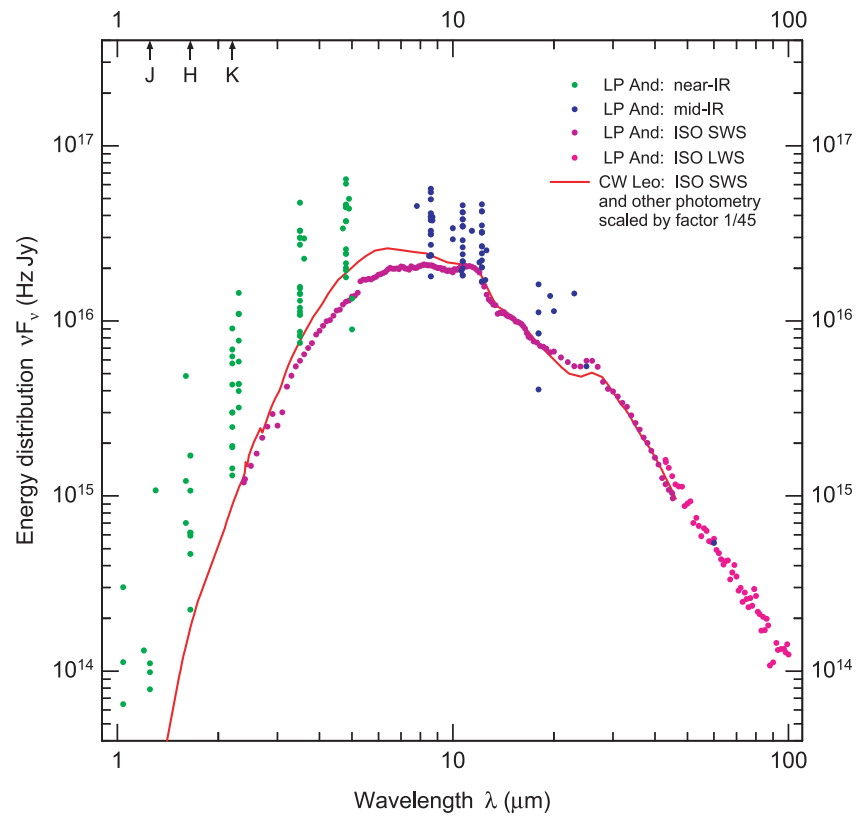

Fig. 3. Observed SEDs of two carbon stars, LP And and CW Leo (IRC +10216$)$, show close similarity. The observations for LP And are shown by the filled circles, and for CW Leo they are shown by the solid line.

\subsection{Model parameters}

We used all the parameters of our model of CW Leo, modifying them slightly, in order to fit the observations of LP And. Since the speckle observations of LP And do not show any discernible deviations from spherical symmetry, we computed mostly spherical models. Nevertheless, to investigate the effects of bipolar cavities similar to those of CW Leo, a number of $2 \mathrm{D}$ models were also computed. In this paper we describe the results of only the simpler spherical models, as a very small amount of spatially-resolved observational constraints do not justify addition of other free parameters.

For the same reason, we simplified the density distribution of the model of LP And compared to that of CW Leo. Models should never include arbitrary structures that are not constrained by observational data; the less data, the simpler our models should remain. Complexity can only be increased when there are sufficiently detailed observational data.

The model parameters are summarized in Table 4. The dust density distribution is assumed to be a broken power law $\rho \propto r^{\alpha_{i}}$, with the exponents $\alpha_{i}(i=1,2,3)$ allowed to change in different radial zones. The mass-loss rate in Table 4 corresponds to the outer envelope ( $r \gtrsim 100 \mathrm{AU}$ ) having a $\rho \propto r^{-2}$ density distribution, and it is based on the adopted outflow velocity of $14 \mathrm{~km} \mathrm{~s}^{-1}$. As usual, in this kind of dust radiative transfer modelling, one obtains only the parameters of the dust envelope directly. All values for the gas component were derived by using the dust-to-gas mass ratio $\rho_{\mathrm{d}} / \rho=0.0039$. We would like to emphasize that not all of the parameters in Table 4 are independent free parameters and that results of our radiative transfer modelling are fully invariant with respect to distance $D$, provided that several parameters are scaled in the following simple way:

$L \propto D^{2}, M \propto D^{2}, \dot{M} \propto D, R \propto D, \rho \propto D^{-1}$.

These relations make it easy to scale the model results to another distance, if necessary.

\subsubsection{Stellar parameters}

As stellar radii normally cannot be directly estimated from observations, our usual approach is to specify two independent parameters $\left(L_{\star}, T_{\star}\right)$ and to compute $R_{\star}$ from the StefanBoltzmann law. We assume that the effective temperature varies around those values that are similar to those of CW Leo, which are typical for stars at the top of the AGB. More than twice larger luminosity amplitude of LP And compared to CW Leo also implies a larger amplitude in the temperature variations. From the known $L_{\star}$ and adopted $T_{\star}$ at the minimum phase, we derived stellar radius $R_{\star}$ at that phase. Since for a pulsating star, it is reasonable to implicitly assume variations of the stellar radius, following our approach for CW Leo (Men'shchikov et al. 2001), we assumed that the radius changes by $20 \%$ between the minimum and maximum of stellar luminosity. Now, having $R_{\star}$ (and also $L_{\star}$ ) at the maximum, we derived the corresponding $T_{\star}$ from the Stefan-Boltzmann law.

With these assumptions for the adopted distance $D=$ $740 \mathrm{pc}$, the luminosity $L_{\star}$ of LP And varies from 16200 to $2900 L_{\odot}$ between the maximum and minimum brightness, with a period of 617 days. The effective temperature $T_{\star}$ of the star changes from 3550 to $2100 \mathrm{~K}$ between the two phases, whereas the resulting stellar radius $R_{\star}$ varies in a relatively narrow range, from 340 to $410 R_{\odot}$. The last values of the temperatures and radii are our reasonable assumptions, as LP And is embedded in an opaque envelope and they cannot be constrained by observations.

Since the low-density, extended, molecular atmospheres of AGB stars can only be described very poorly by a blackbody, we used a much more accurate description of the stellar SED provided by the models of stellar atmospheres. As in the modelling of CW Leo, we used the models computed for us by Rita Loidl with the MARCS code (Gustafsson et al. 1975) in the version of Jørgensen et al. (1992). One of the stellar SEDs (for $\phi=0.64)$ is shown in Fig. 4.

\subsubsection{Density distribution}

The density distribution of the model envelope is described by a broken power law, $\rho \propto r^{\alpha}$. The envelope is subdivided 


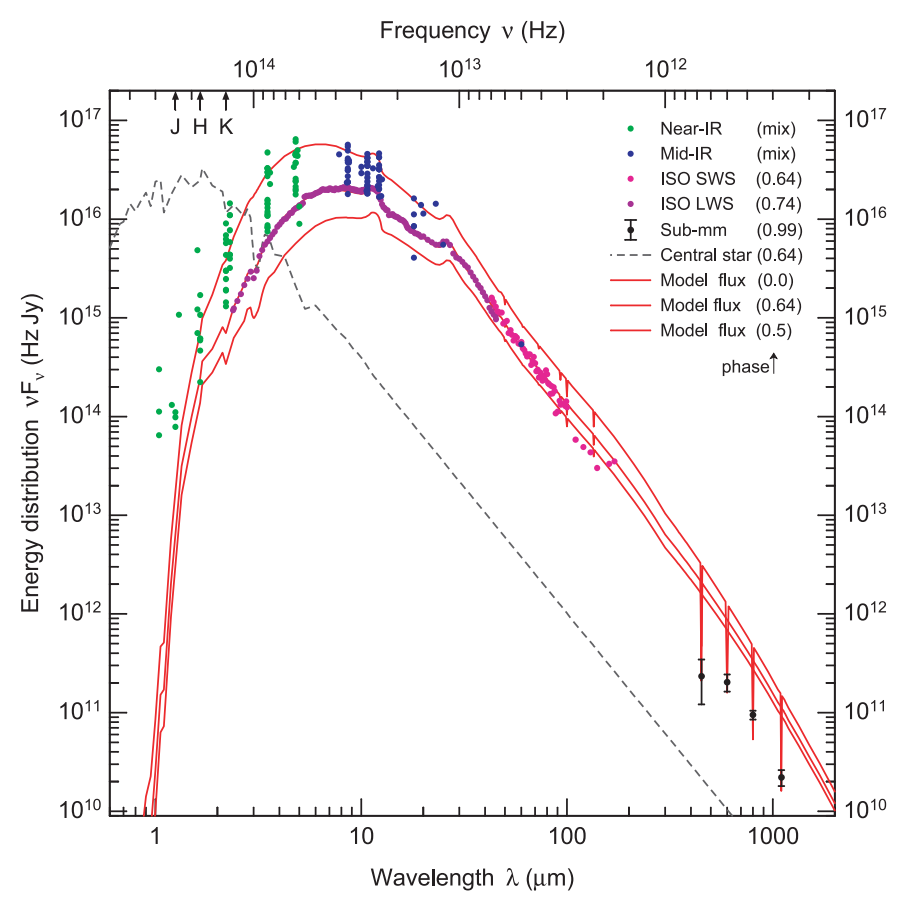

Fig. 4. Observed SED of LP And compared to our model at three different phases $(\phi=0.0,0.64$, and 0.5$)$. Stellar continuum (at $\phi=0.64)$ and total and beam-matched fluxes are plotted. If available, error bars for the fluxes are drawn whenever they are larger than the symbols. Effect of beam sizes is shown by the vertical lines, clearly visible in the model SEDs at $\lambda>400 \mu \mathrm{m}$. The (sub)millimeter fluxes were measured at almost exactly the maximum stellar luminosity. The vertical lines at the four wavelengths show the range of the total model flux correction for the finite (small) beam sizes used in the observations. Only the lower points of the vertical lines are relevant, however we have connected them to the adjacent continuum by straight lines, to illustrate the effect better. The beam-matched fluxes at $\lambda<10 \mu \mathrm{m}$ include both the effect of $16^{\prime \prime}$ beams and interstellar extinction. The three arrows at the top axis indicate the central frequencies of the $J, H$, and $K$ photometric bands.

into several shells, each with a fixed power-law exponent $\alpha$. As we have fewer observational constraints for LP And compared to CW Leo, in this model we simplified the density profile by using only three power-law exponents $\left(\alpha_{1}, \alpha_{2}, \alpha_{3}\right.$, see Table 4, Fig. 5). The broken-power-law density profile may result from a complex interplay between stellar pulsations, changing mass-loss rate, and radiative acceleration of dust and gas in the innermost parts of the envelope. The outer envelope's density profile $\left(\rho \propto r^{-2}, r>80 \mathrm{AU}\right)$ is produced by a constant-velocity spherical outflow with a constant mass-loss rate $\dot{M}=1.9 \times 10^{-5} M_{\odot} \mathrm{yr}^{-1}$. The total mass of the envelope is $M=3.2 M_{\odot}$ within its outer radius $R_{2}=2.9 \mathrm{pc}$, almost the same as in CW Leo. The mass is based on the same value of $\rho_{\mathrm{d}} / \rho=0.0039$ in the outer envelope and the same dust model as in Men'shchikov et al. (2001). These assumptions are justified by the fact that the observed infrared emission bands are extremely similar in both LP And and CW Leo.

\subsubsection{Dust model}

Different dust components exist in the envelope of LP And everywhere from the stellar photosphere to the outer boundary, producing the total visual optical depth $\tau_{V} \approx 25$ towards the star. As in CW Leo, both the radial distribution and composition of dust in our model of LP And are strongly inhomogeneous. The emission feature at $11.3 \mu \mathrm{m}$ was best reproduced by Men'shchikov et al. (2001) by [SiC, C] particles, which are unorganized aggregates made of incompletely amorphous carbon grains with significant graphitic content and silicon carbide grains. The inhomogeneous material was modelled with volume fractions of $15 \%$ for $\alpha$-SiC (Choyke \& Palik 1985) and of $85 \%$ for the carbon material cel800 (Jäger et al. 1998). The broad emission feature between 24 and $37 \mu \mathrm{m}$ was best reproduced by $[\mathrm{SiC}, \mathrm{C}]$ cores covered by a thin layer of magnesiumiron sulfides $\left[\mathrm{Mg}_{0.5} \mathrm{Fe}_{0.5}\right] \mathrm{S}$ (referred to as $[\mathrm{SiC}, \mathrm{C}]+[\mathrm{Mg}, \mathrm{Fe}] \mathrm{S}$ grains).

The abundances of the dust components and the grain size distribution $\mathrm{d} n / \mathrm{d} a \propto a^{\gamma} \exp \left(-a / a_{\exp }\right)$ in our model depend on the distance from the star. The dust-to-gas mass ratios $\rho_{\mathrm{d}} / \rho$, material densities $\rho_{\mathrm{gr}}$, limiting grain radii $a_{\mathrm{min}}$ and $a_{\mathrm{exp}}$, and exponents $\gamma$ of the size distribution for the three dust components are all the same as in Table 2 of Men'shchikov et al. (2001). The only difference is that the transition to larger outer dust grains occurs at $r=80 \mathrm{AU}$ in this model of LP And, instead of $100 \mathrm{AU}$ in CW Leo.

The interstellar (IS) dust extinction to this relatively distant star was taken into account by reddening the model fluxes using the analytic fits by Cardelli et al. (1989) extended to $\lambda=100 \mu \mathrm{m}$ with the data from Savage \& Mathis (1979) and parameters $R_{V}=3.1$ and $n_{\mathrm{H}}=1 \mathrm{~cm}^{-3}$. As has been shown by modelling other objects, such as the Red Rectangle and RY Sct (Men'shchikov et al. 2002b; Men'shchikov \& Miroshnichenko 2005), accounting for the IS extinction by accurate fitting of the optical and ultraviolet observations may be instrumental in the derivation of distances and physical parameters of objects. In the case of LP And, however, the absence of the optical and ultraviolet photometry has not allowed our modelling to place better constraints on the amount of IS reddening and the distance towards the star. From our modelling of LP And, it is clear that the IS extinction plays a minor role in shaping of its SED.

\subsection{Spectral energy distribution}

Figure 4 compares model energy distributions with the observed SEDs of LP And at three phases, $\phi=0.0,0.64$, and 0.5. We compare the model SEDs to the observed spectrophotometry and broad-band flux distribution of LP And in the entire range from optical to millimeter wavelengths. Since the central energy source is a pulsating star, its luminosity, effective temperature, and radius are also changing with the period $P=617$ days (Sect. 2.1).

The effect of beam sizes is shown in Fig. 4 at all wavelengths where fluxes were measured; it is visible mainly at the far-IR and (sub)millimeter wavelengths. The beam-matched fluxes are lower by an order of magnitude than the total model 
Table 4. Physical parameters of our model of LP And.

\begin{tabular}{lllcl}
\hline \hline Parameter & Symbol & Units & Value & Comment \\
\hline Distance & $D$ & $\mathrm{pc}$ & 740 & estimated (Sect. 2.4) \\
Maximum luminosity & $L_{\star}$ & $L_{\odot}$ & 16200 & estimated from SED (Sects. 2.3, 2.4) \\
Minimum luminosity & $L_{\star}$ & $L_{\odot}$ & 2900 & estimated from SED (Sects. 2.3, 2.4) \\
Eff. temperature (max) & $T_{\star}$ & $\mathrm{K}$ & 3550 & derived $\left(T_{\star}=(4 \pi \sigma)^{-1 / 4} L_{\star}^{1 / 4} R_{\star}^{-1 / 2}\right)($ Sect. 3.1.1) \\
Eff. temperature (min) & $T_{\star}$ & $\mathrm{K}$ & 2100 & assumed (Sect. 3.1.1) \\
Stellar radius (max) & $R_{\star}$ & $R_{\odot}$ & 340 & assumed $\left(20 \%\right.$ change in $\left.R_{\star}\right)$ (Sect. 3.1.1) \\
Stellar radius (min) & $R_{\star}$ & $R_{\odot}$ & 410 & derived $\left(R_{\star}=(4 \pi \sigma)^{-1 / 2} L_{\star}^{1 / 2} T_{\star}^{-2}\right)($ Sect. 3.1.1) \\
Inner boundary & $R_{1}$ & $\mathrm{AU}$ & 3.3 & derived $\left(R_{1} \approx 2 R_{\star}\right)$ \\
Outer boundary & $R_{2}$ & $\mathrm{AU}$ & $6 \times 10^{5}$ & derived $\left(R_{2} \approx 3\right.$ pc; from $\left.\rho\left(R_{2}\right) \approx \rho_{\mathrm{ISM}}\right)$ \\
Envelope's total mass & $M$ & $M_{\odot}$ & 3.2 & derived (from the adopted $\left.\rho_{\mathrm{d}} / \rho=0.0039\right)$ \\
Initial outflow velocity & $v$ & $\mathrm{~km} \mathrm{~s}^{-1}$ & 4.4 & derived (from the model density distribution) \\
Outflow velocity & $v$ & $\mathrm{~km} \mathrm{~s}^{-1}$ & 14.0 & assumed (from the literature, Sect. 1) \\
Mass-loss rate & $\dot{M}$ & $M_{\odot} \mathrm{yr}^{-1}$ & $1.9 \times 10^{-5}$ & derived (from $v$ and $\rho(r)$ at $r>80 \mathrm{AU})$ \\
Dust density exponent & $\alpha_{1}$ & - & +1 & model $(3.3-45 \mathrm{AU})$ \\
Dust density exponent & $\alpha_{2}$ & - & -4 & model $(45-80 \mathrm{AU})$ \\
Dust density exponent & $\alpha_{3}$ & - & -2 & model $\left(80-6 \times 10^{5}\right.$ AU) \\
\hline
\end{tabular}

fluxes, emphasizing the very large extent of the envelope, which is much larger than the $18.5^{\prime \prime}$ photometric apertures used in the observations. Note that care must be taken to compare our model with only those measurements, which were obtained close to the luminosity phase considered. At $\lambda \lesssim 100 \mu \mathrm{m}$, the dust radiation is compact enough compared to the beam sizes of the observations so there is no significant beam effect there.

The model SED at $\phi=0.64$ fits the entire ISO spectrum perfectly, including the two broad emission bands and the photospheric absorption feature at $\lambda \approx 3 \mu \mathrm{m}$, serving as a reference for our discussion of the SEDs at the other two phases, where no such good photometry exists (Fig. 4). The SED at maximum luminosity fits the (sub)millimeter fluxes quite well, especially at $450-600 \mu \mathrm{m}$. The observed fluxes at $800 \mu \mathrm{m}$ and $1.1 \mathrm{~mm}$ seem to be slightly above the model predictions, which might indicate a somewhat shallower long-wavelength grain emissivity in LP And than the one adopted in our model $\left(Q_{\mathrm{abs}} \propto \lambda^{-1}\right)$, possibly due to a larger maximum grain size.

The model SED at $\phi=0.0$ fits existing mid-IR fluxes; however, at $\lambda \lesssim 5 \mu \mathrm{m}$ it starts to deviate noticeably from the scattered observations (Fig. 4). This may be an indication of a moderate departure from spherical symmetry in the envelope, similar to but smaller than in CW Leo. We investigated this idea by computing several $2 \mathrm{D}$ radiative transfer models of the envelope with bipolar cavities, similar to the ones in CW Leo. As expected, opening such cavities with a lower density enhances the near-IR fluxes and makes the model agree much better with the higher observed fluxes at $\phi=0.0$.

Although the model with bipolar cavities might work, we do not present it here as our model of LP And, because our speckle observations do not show noticeable deviations from spherical symmetry. One would need conclusive observational evidence of deviations from spherical geometry and spatially-resolved images before any 2D model (with several additional free parameters) could be justified (see also Sect. 3.4). Reasonable models must strike a balance between the number of free parameters and the number of observational constraints. In contrast to CW Leo, the envelope of LP And has been observed less.

On the other hand, it may simply be the long-term nonperiodic changes in the envelope of LP And that are responsible for the higher near-IR fluxes. We cannot discard the possibility that the envelope was somewhat less opaque three decades ago. In fact, the rapid evolution observed in the similar envelope of CW Leo suggests that variability on such a time scale may have occurred in LP And, too. There may also be a number of other possible explanations for this discrepancy at short wavelengths, such as the absence of molecular opacity in our model or the hydrostatic model atmospheres employed for this pulsating star.

Although the model SED at $\phi=0.5$ has no observational counterpart, it does illustrate how the energy distribution changes with decreasing luminosity (Fig. 4). Decreasing grain temperatures in the dust formation zones lead to a significantly decreasing contribution of thermal emission at the near- and mid-IR wavelengths, whereas the dust scattering remains unaffected. This leads to an even more pronounced appearance of the absorption features at $\lambda \approx 2.2$ and $3 \mu \mathrm{m}$ in the model SED, which are the absorption bands from the stellar spectrum, scattered by the envelope. They almost disappear at $\phi=0.0$ due to the overwhelming emission of the significantly hotter dust.

\subsection{Densities and temperatures}

In Fig. 5 we display the density and temperature distributions in the model of LP And for only small grains with a radius $a=0.01 \mu \mathrm{m}$. In the immediate vicinity of the star $\left(\theta \lesssim 0.06^{\prime \prime}\right.$, $r \lesssim 45 \mathrm{AU}$ ), we assumed a $\rho_{\mathrm{d}} \propto r$ gradient of the dust density, 


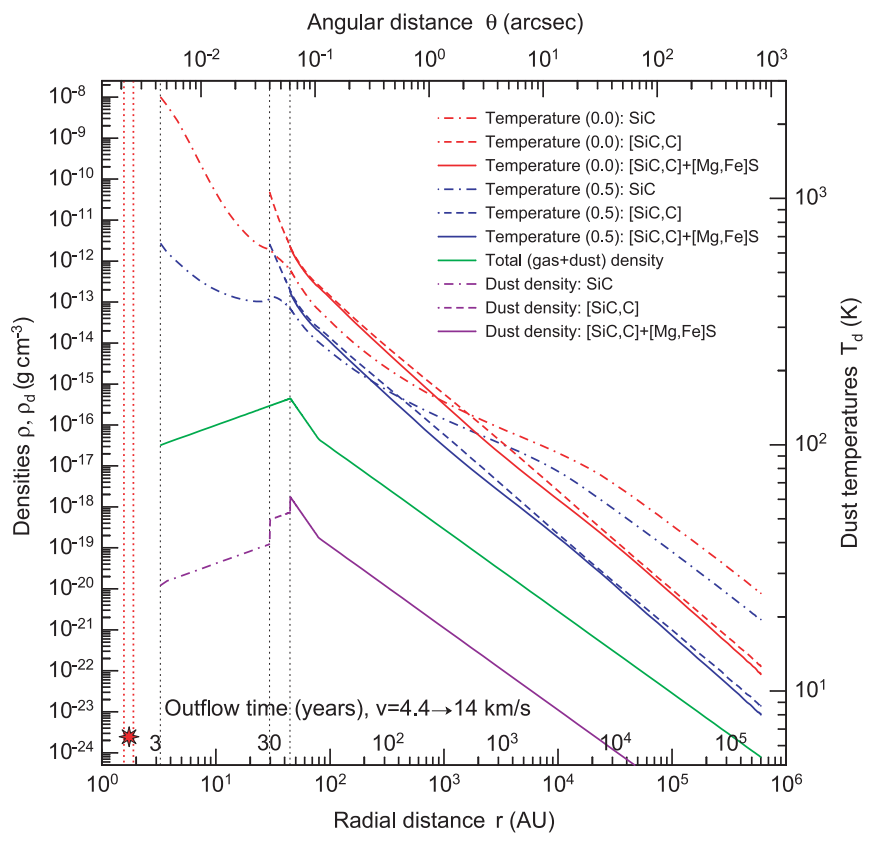

Fig. 5. Densities and temperatures of dust grains in our model of the envelope of LP And. Dust densities and temperatures are displayed for the smallest grains of the respective size distributions. Dust temperatures are shown for the maximum $(\phi=0.0)$ and minimum $(\phi=0.5)$ stellar brightness (the upper and lower sets of curves, respectively). Also shown are the individual dust density profiles for the most abundant component at the given distance and the total density distribution. The latter has the shape of the dust density and is shown only for an illustration of what it would be if $\rho_{\mathrm{d}} / \rho$ were constant, as assumed in our model. Three dotted vertical lines indicate the formation zones of the dust components. The other dotted vertical lines on the left side of the plots, marked with an asterisk, show stellar radii at the maximum and minimum luminosity phases. Additional labelling above the lower abscissa shows expansion times for constant outflow velocities of $v=4.4 \mathrm{~km} \mathrm{~s}^{-1}(r<45 \mathrm{AU})$ and $v=14 \mathrm{~km} \mathrm{~s}^{-1}(r>45 \mathrm{AU})$.

as done by Men'shchikov et al. (2001) for CW Leo. Since there are no observational constraints on the radial dependence of the dust-to-gas mass ratio, we have no other choice than to assume that $\rho_{\mathrm{d}} / \rho$ is constant there. This implies that our model's "gas density" $\rho$ is also proportional to $r$ (Fig. 5).

The gas density distribution close to the star is neither observationally constrained nor predicted by the model. The situation in the innermost parts of the envelope is very complicated due to both stellar pulsations and unknown details of the temporal and radial dependences of the dust formation process there. Shock waves, periodic dust formation episodes, and changing mass-loss rate around the pulsating star can all lead to a non-monotonic gas density distribution. However, we emphasize that the gas component is not included in our model at all, and the gas density profile was obtained from the dust density using the dust-to-gas mass ratio and is shown in Fig. 5 for illustration only. Both our observations and model do not resolve the innermost parts of the envelope with $\theta \lesssim 0.3^{\prime \prime}$ ( $r \lessgtr 10 R_{\star} \approx 20 \mathrm{AU}$ ), and the model cannot predict the gas density distribution there. In reality, $\rho_{\mathrm{d}} / \rho$ is a function of $r$, and it may change a lot due to condensation of new dust components out of the gas phase. Although the gas density decreases with distance from the star in a stationary wind, the dust density increases with $r$ in the envelope's parts where dust formation of various dust components takes place.

We computed several models varying dust density profile in these inner regions of the envelope. The only constraint from the modelling is that the inner $45 \mathrm{AU}$ from the star should be relatively optically thin. Thus, the density profile could have any form, from very steeply rising with distance to constant density. However, dust density cannot decrease with distance, otherwise the optical depth would be high enough to alter the model SED and visibilities. In addition, the dust-formation zone of our model (3-45 AU) can be thinner (e.g., 20-45 AU), but this does not influence the model results.

In the outer envelope of LP And ( $\left.\theta \gtrsim 0.1^{\prime \prime}, r \gtrsim 80 \mathrm{AU}\right)$, we adopted the standard $\rho \propto r^{-2}$ profile. Since there are no spatially-resolved observational constraints for the outer envelope (unlike in CW Leo), we simplified the density profile by using just the single power-law exponent, which implies a spherical outflow with a constant velocity and mass-loss rate. The gas density at $R_{2}=6 \times 10^{5} \mathrm{AU}$ in our model is very low $\left(n_{\mathrm{H}} \approx 0.5 \mathrm{~cm}^{-3}\right.$ ), on the order of the average density of the interstellar medium. The envelope's outer radius, gas density, and mass $M=3.2 M_{\odot}$ are the same as in the model of CW Leo (Men'shchikov et al. 2001).

The temperature profiles in Fig. 5 reflect different temperatures of the dust components, according to the optical properties of the grains. In particular, the $\mathrm{SiC}$ dust with its strong absorption feature at $10.75 \mu \mathrm{m}$ is significantly cooler than the other components in the innermost parts of the envelope, producing a 50\% jump between their temperature profiles at $r=$ $30 \mathrm{AU}$. The same $\mathrm{SiC}$ grains are warmer by a factor of $2-2.5$ than the other components in the outermost regions of the envelope of LP And. This is because the reprocessed (cooler) radiation field there peaks in the mid-IR, so more radiation can be absorbed by the strong $\mathrm{SiC}$ dust feature.

Two vertical jumps in the density profiles of individual dust components at $r=30$ and $45 \mathrm{AU}$, appearing in Fig. 5 on top of the broad density peak described above, are due to the condensation of $\mathrm{C}$ and $\left[\mathrm{Mg}_{0.5} \mathrm{Fe}_{0.5}\right] \mathrm{S}$, correspondingly, from the gas phase. Our model assumes that almost all $\mathrm{SiC}$ grains are incorporated into the $[\mathrm{SiC}, \mathrm{C}]$ aggregates at $r \approx 30 \mathrm{AU}$, which are in turn almost entirely incorporated into the $[\mathrm{SiC}, \mathrm{C}]+[\mathrm{Mg}, \mathrm{Fe}] \mathrm{S}$ grains at $r \approx 45 \mathrm{AU}$. In our model of LP And, the dust-formation radii do not change with stellar luminosity phase (Fig. 5). This assumption is based on the results of the detailed modelling of CW Leo (see Men'shchikov et al. 2001, 2002a) and on the absence of any observational data for LP And that would indicate otherwise.

The dust model is consistent with the picture of dust formation in the winds of carbon stars. In fact, the SiC dust, made of very refractive material, forms very close to the stellar photosphere, where the newly formed small $\mathrm{SiC}$ grains have temperatures $T_{\mathrm{SiC}} \approx 2000 \mathrm{~K}$. Those small dust grains can serve as condensation sites for the formation of the most abundant but less refractive carbon materials when the wind reaches greater distances and lower temperatures $T_{\mathrm{C}} \approx 1000 \mathrm{~K}$, leading eventually to the formation of the $[\mathrm{SiC}, \mathrm{C}]$ aggregates. Even less refractive 
materials, such as the magnesium-iron sulfides, can later create mantles on those inhomogeneous grains at $T_{[\mathrm{Mg}, \mathrm{Fe}] \mathrm{S}} \approx 600 \mathrm{~K}$.

The time-dependent mass-loss rate $\dot{M}$ of LP And can be derived readily from the density distribution of our model, assuming a uniform velocity of $v=14 \mathrm{~km} \mathrm{~s}^{-1}$ and assuming that the dust-to-gas mass ratio $\rho_{\mathrm{d}} / \rho$ in the envelope does not change with time. Our model's mass-loss rate $\dot{M} \approx 1.9 \times 10^{-5} M_{\odot} \mathrm{yr}^{-1}$ in the old wind (Table 4 ) is in good agreement with the recent CO line observations of the outer envelope (Schöier \& Olofsson 2001). Within the framework of the stationary spherical wind, the density peak at $r=45 \mathrm{AU}$ (Fig. 5) would imply that either $\dot{M}$ (or $\rho_{\mathrm{d}} / \rho$ ) is higher on the $\sim 0.1^{\prime \prime}$ scales or the outflow velocity is lower there, by a factor of $\sim 3$. Most natural is to assume that an acceleration of the wind from 4.4 to $14 \mathrm{~km} \mathrm{~s}^{-1}$ by the radiation pressure on the newly formed dust grains creates the density peak and the steeper $\rho \propto r^{-4}$ density profile in our model at $45<r<80 \mathrm{AU}$, although one cannot exclude that $\dot{M}$ or $\rho_{\mathrm{d}} / \rho$ are higher now than they were a few decades ago. Note that there are no such indications for LP And, in contrast to our detailed speckle observations of CW Leo that suggested a time-dependent, increasing mass-loss rate (Men'shchikov et al. 2002a).

\subsection{Near-infrared visibilities}

The model of LP And can also be compared in terms of the near-IR visibilities from our speckle observations in the $H$ and $K^{\prime}$ bands. A 2D Fourier transform of the model intensity map gives a much more sensitive measure of the intensity distribution over all spatial frequencies than do the beam-matched SEDs presented in Fig. 4.

The azimuthally-averaged $H$ - and $K^{\prime}$-band visibilities in Fig. 6 display noticeably different shapes. The visibility drops faster at low frequencies and flattens out at intermediate frequencies, compared to the almost linear decrease in the $K^{\prime}$-band visibilities. The $H$-band visibility shape suggests a combination of predominantly scattering at large scales (low frequencies) and a $\sim 60 \%$ contribution from an unresolved source, the latter being obviously the central star. There is a good general agreement with the model visibility in the $H$ band. It is not very likely that the steep drop in the visibilities at high spatial frequencies (above $12.5 \mathrm{arcsec}^{-1}$ ) is an artefact from the observations, although its nature is not completely clear. Possibly, the uncertainties of the data at the frequencies approaching the diffraction cut-off were higher than those indicated by the error bars, or this is an effect of some deviations from spherical geometry, as in CW Leo; for the purpose of this discussion we ignore those parts of the curves.

The $K^{\prime}$-band model visibility in Fig. 6 differs from the observed one significantly. The model diplays a more resolved intensity distribution, with by a factor of 3 lower visibilities. Our modelling of LP And indicated that this is caused by a larger contribution from the resolved hot dust emission and a smaller relative contribution from the star at this longer wavelength. An investigation of the model parameter space showed that it is very difficult to fit the SED and both visibilities. In fact, we have been unable to find any model that fits all those

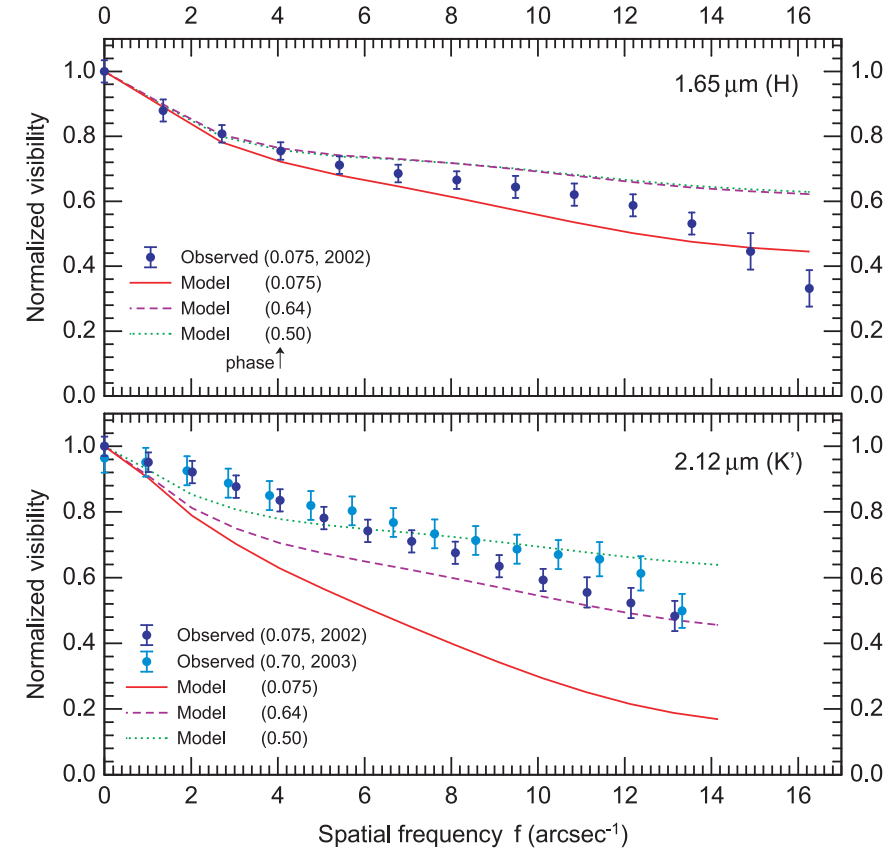

Fig. 6. Model visibilities of LP And in the $H$ and $K^{\prime}$ bands (phases $\phi=0.08,0.64$, and 0.50 ) are compared to the azimuthally-averaged visibilities from our speckle-interferometry data obtained in 2002 and 2003 (phases $\phi \approx 0.08$ and 0.70 , respectively).

observational constraints well enough at the correct phase $\phi=$ 0.08 . Although the SED and $H$ visibility could be reproduced very well, the model $K^{\prime}$-band visibility was almost always lower than the observed one by at least a factor of 2 .

There may be several reasons for the larger difference of the model visibilities at the two wavelengths compared to the observed ones. We cannot exclude the possibility that the stellar continuum of LP And in the $H$ and $K^{\prime}$ bands differs from that given by the hydrostatic model atmospheres adopted in the model. One can easily see from Fig. 4 that the stellar continuum differs between the wavelengths by a factor of $\sim 3$. If, in reality, the stellar flux in the $K^{\prime}$ band were almost the same as that in $H$, then the increased contribution from the star in the $K^{\prime}$ band would bring the model visibility up to the observations. It is also conceivable that the wavelength-dependent optical properties of grains in the envelope may deviate in this near-IR region from those assumed in our dust model. If the excessive dust emission or scattering in the $K^{\prime}$ band were somehow reduced by a factor of $\sim 3$, the corresponding visibility profile would fit the observed one. The envelope's structure might also be different: a steep density (optical depth) gradient would reduce the difference between the $H$ and $K^{\prime}$ visibilities. However, such a structure would also tend to degrade the model SED. There may also be significant molecular opacity in the envelope of LP And, which is not included in our models. It may well be that a combination of the above factors might explain the discrepancy in the model visibilities.

One of the additional explanations may be moderate deviations from spherical geometry that are not as large as in CW Leo and thus not noticeable in our speckle-interferometry observations. In fact, we computed several 2D models of 
LP And with the same geometry of bipolar cavities as in CW Leo (Men'shchikov et al. 2001) and a reduced density contrast. They showed a trend of both visibilities towards each other, together with the enhanced near-IR fluxes necessary to fit the observed SED of LP And better at maximum luminosity (Sect. 3.2). In order for that geometry to work, we should observe the object through the polar regions of a somewhat lower optical depth. We did not fully explore the 2D models of LP And in this work, as with the insufficient amount of the observational constraints and no clear evidence of the deviations from spherical geometry, they would not be justified.

Our model fails to reproduce one of the two visibilities from our speckle observations, but it is not the only observational data. The visibilities cannot be considered the most important data used in the modelling. There is plenty of observational information and other constraints for the modelling in the well-defined SED of LP And. The fact that the model is fully consistent with the entire SED of the star - from the near-IR to millimeter wavelengths, including the shapes of two dust emission bands at 11 and $27 \mu \mathrm{m}$, the absorption feature visible in the stellar continuum at $3 \mu \mathrm{m}$, and the effect of beam sizes at submillimeter wavelengths, as well as the $H$-band visibility profile - demonstrates that the model is sufficiently realistic.

\section{Evolutionary status}

It is not easy to draw some definite conclusions on the evolutionary status of an evolved star based only on the results of radiative transfer modelling. The physical parameters we derived in this work are consistent with the central star being a rather highly-evolved AGB star of an intermediate initial mass. This can be concluded from the following facts. ( $i)$ The massloss rates in excess of $10^{-5} M_{\odot} \mathrm{yr}^{-1}$ imply highly-evolved stars. (ii) The star is a carbon star of a moderate luminosity, which indicates that the hot-bottom burning (HBB) is not playing a dominant role. Stars with higher initial masses would undergo hot-bottom burning and thus must have significantly higher luminosities and also destroy carbon in their outer convective layers. (iii) The star's envelope chemistry implies a star of an intermediate initial mass.

The envelope composition is very important in determining the evolutionary status and initial masses of the stars. There are some measurements of the isotopic ratios ${ }^{12} \mathrm{C} /{ }^{13} \mathrm{C}$ and ${ }^{14} \mathrm{~N} /{ }^{15} \mathrm{~N}$ in LP And (Wannier et al. 1991; Greaves \& Holland 1997) indicating some very mild HBB. This would be in line with an initial mass close to $4 M_{\odot}$, approximately the limiting initial mass for the onset of $\mathrm{HBB}$. The two isotopic ratios measured for LP And are ${ }^{12} \mathrm{C} /{ }^{13} \mathrm{C}=15-45$ and ${ }^{14} \mathrm{~N} /{ }^{15} \mathrm{~N}>550$. The rather low ${ }^{12} \mathrm{C} /{ }^{13} \mathrm{C}$ ratio indicates the onset of $\mathrm{HBB}$, but the ratio is still clearly above the equilibrium value of $\mathrm{CNO}$ cycling $(\sim 3-4)$. The ${ }^{14} \mathrm{~N} /{ }^{15} \mathrm{~N}$ ratio might already be enhanced, but apparently values $\sim 2 \times 10^{5}$ have been measured. Thus, LP And shows only mild HBB and therefore, the initial stellar mass should not be larger than $4 M_{\odot}$, which is consistent with the rather moderate luminosities. On the other hand, the initial mass of the star should also not be too small. Otherwise, there would be absolutely no indication of $\mathrm{HBB}$ (higher ${ }^{12} \mathrm{C} /{ }^{13} \mathrm{C}$ ) and there would be inconsistency with the mass $M=3.2 M_{\odot}$ of the dusty envelope derived in this work. A carbon star like LP And with an initial mass of $4 M_{\odot}$ has a core mass of $\sim 0.8 M_{\odot}$, and the dusty envelope's mass is consistent with the values. This initial mass would agree well with a star that is really close to the end of its AGB evolution. The possible onset of the deviations of the dusty envelope from spherical geometry (or clumpiness) can also be interpreted as pointing in the same direction.

\section{Conclusions}

This paper presents our near-infrared speckle interferometry of the carbon star LP And in the $H$ and $K^{\prime}$ bands with diffractionlimited resolutions of 56 and 72 mas, new photometry in the $J$, $H, K, L$, and $M$ bands, and the results of the first detailed radiative transfer modelling of its dusty envelope. The reconstructed $2 \mathrm{D}$ visibility revealed no significant deviations in the envelope from spherical symmetry.

Instead of performing an extensive (and time-consuming) exploration of the model parameter space from scratch, we based the model on the results of the detailed model of the beststudied carbon star, CW Leo, presented by Men'shchikov et al. (2001, 2002a). This was justified by the fact that many properties of both stars and their envelopes are extremely similar. Our attempts to obtain accurate, self-consistent fits of all existing observational data for LP And allowed us to understand basic physical properties of the object with better accuracy than ever before. We found that, in fact, both the spectral energy distribution and the $H$-band visibility of LP And can be reproduced by a spherical envelope with parameters that are very similar to those of CW Leo. We find that our $K^{\prime}$-band visibility could not be fitted by our spherical model. There may be several possible reasons of this interesting result, so more observations are required to determine what actually causes this effect.

From our analysis of all observations of LP And, we estimated its new pulsation period as $P=617 \pm 6$ days and distance $D=740 \pm 100 \mathrm{pc}$. The model suggests that this variable star has luminosity $L_{\star} \approx 16200-2900 L_{\odot}$, effective temperature $T_{\star} \approx 3550-2100 \mathrm{~K}$, and radius $R_{\star} \approx 340-410 R_{\odot}$. In contrast to the luminosities, the effective temperatures and radii of LP And are not very well constrained by the modelling, as the envelope is opaque ( $\tau_{V} \approx 25$ in the visible). The star is losing its mass at the rate of $\dot{M} \approx 1.9 \times 10^{-5} M_{\odot} \mathrm{yr}^{-1}$, assuming that the wind is accelerated from $v=4.4 \mathrm{~km} \mathrm{~s}^{-1}$ to the observed velocity $v=14 \mathrm{~km} \mathrm{~s}^{-1}$ just outside the dust formation zone at $r \approx 45 \mathrm{AU}$. The dusty envelope of LP And extends to distances $R_{2} \approx 3$ pc from the star. The total mass of the envelope lost by the central star is $M=3.2 M_{\odot}$, assuming the same dust-to-gas mass ratio $\rho_{\mathrm{d}} / \rho=0.0039$ as in CW Leo. The initial mass of the star is $M_{\star}^{0} \approx 4 M_{\odot}$ and its present-day core mass is $\sim 0.8 M_{\odot}$.

In addition to $\mathrm{SiC}$ dust, the model contains inhomogeneous grains made of a mixture of $\mathrm{SiC}$ and incompletely amorphous carbon with thin $\left[\mathrm{Mg}_{0.5} \mathrm{Fe}_{0.5}\right] \mathrm{S}$ mantles. This same dust model fits the $11.3 \mu \mathrm{m}$ and $27 \mu \mathrm{m}$ emission bands extremely well in both LP And and CW Leo. This confirms the idea that the model can be successfully applied for a number of carbon stars with similar properties (Men'shchikov et al. 2001). Although there are no such indications from the existing observational 
data, our modelling of LP And cannot exclude that there may be slight deviations from spherical geometry in its envelope (such as bipolar cavities, clumpiness, or inhomogeneities), suggesting that the star's evolutionary stage is also very similar to that of CW Leo.

Acknowledgements. A.B.M. acknowledges support from the Natural Sciences and Engineering Research Council of Canada (NSERC). The authors thank the anonymous referee for useful comments.

\section{References}

Cardelli, J. A., Clayton, G. C., \& Mathis, J. S. 1989, ApJ, 345, 245

Choyke, W. J., \& Palik, E. D. 1985, in Handbook of Optical Constants of Solids, ed. E. D. Palik (Academic Press), 587

Cohen, M. 1979, MNRAS, 186, 837

Cohen, M. 1984, MNRAS, 206, 137

Cohen, M., \& Hitchon, K. 1996, AJ, 111, 962

Gezari, D. Y., Pitts, P. S., \& Schmitz, M. 1999, Catalog of Infrared Observations, Edition 5

Greaves, J. S., \& Holland, W. S. 1997, A\&A, 327, 342

Groenewegen, M. A. T., de Jong, T., \& Baas, F. 1993, A\&AS, 101, 513

Groenewegen, M. A. T., \& Whitelock, P. A. 1996, MNRAS, 281, 1347

Gullixson, C., Gehrz, R. D., Hackwell, J. A., Grasdalen, G. L., \& Castelaz, M. 1983, ApJS, 53, 413
Gustafsson, B., Bell, R. A., Eriksson, K., \& Nordlund, A. 1975, A\&A, 42, 407

Jäger, C., Mutschke, H., \& Henning, T. 1998, A\&A, 332, 291

Jones, T. J., Bryja, C. O., Gehrz, R. D., et al. 1990, ApJS, 74, 785

Jørgensen, U. G., Johnson, H. R., \& Nordlund, A. 1992, A\&A, 261, 263

Labeyrie, C. D. 1970, A\&A, 6, 85

Lindqvist, M., Schöier, F. L., Lucas, R., \& Olofsson, H. 2000, A\&A, 361, 1036

Lockwood, G. W. 1974, ApJ, 192, 113

Margulis, M., van Berkom, D. J., Snell, R. L., \& Kleinmann, S. G. 1990, ApJ, 361, 673

Men'shchikov, A. B., \& Henning, T. 1997, A\&A, 318, 879

Men'shchikov, A. B., \& Miroshnichenko, A. S. 2005, A\&A, 443, 211

Men'shchikov, A. B., Balega, Y., Blöcker, T., Osterbart, R., \& Weigelt, G. 2001, A\&A, 368, 497

Men'shchikov, A. B., Hofmann, K.-H., \& Weigelt, G. 2002a, A\&A, 392, 921

Men'shchikov, A. B., Schertl, D., Tuthill, P. G., Weigelt, G., \& Yungelson, L. R. 2002b, A\&A, 393, 867

Savage, B. D., \& Mathis, J. S. 1979, ARA\&A, 17, 73

Schöier, F. L., \& Olofsson, H. 2001, A\&A, 368, 969

Simon, T. 1974, AJ, 79, 1054

Strecker, D. W., \& Ney, E. P. 1974, AJ, 79, 797

Wannier, P. G., Andersson, B.-G., Olofsson, H., Ukita, N., \& Young, K. 1991, ApJ, 380, 593

Weigelt, G., Balega, Y., Blöcker, T., et al. 2002, A\&A, 392, 131 